Artigo / Article

\title{
Classificações morfológicas das síndromes mielodisplásicas: da classificação Franco-Americana-Britânica (FAB) à classificação da Organização Mundial da Saúde (OMS)

\author{
Morphologic classifications of myelodysplastic syndromes: from FAB to WHO
}

Teresa C. Bortolheiro

\section{Introdução}

As síndromes mielodisplásicas (SMD) agrupam doenças de espectro clínico intensamente variável, que podem servir de modelo tanto para neoplasias extremamente indolentes quanto para outras de curso clínico muito agressivo., ${ }^{1,2}$ A morbidade e mortalidade da doença estão relacionadas às citopenias periféricas, à hemopoese ineficaz e à evolução para leucemia aguda. A SMD pode surgir como doença primária (de novo) ou secundária, após tratamento quimioterápico, ou radioterápico, para outras neoplasias. ${ }^{1}$ Existem atualmente dois sistemas de classificação para as SMD e grande contro- vérsia sobre qual o mais adequado para caracterizar esse grupo de doenças tão heterogêneas.

\section{A classificação FAB}

Sem dúvida, o surgimento da classificação proposta pelo grupo Franco-Americano-Britânico (FAB) em $1982^{3} \mathrm{e}$ modificada em $1985^{4}$ (Tabela 1) trouxe grandes progressos para a melhor compreensão das SMD. A classificação FAB baseia-se nas alterações morfológicas observadas nas SMD, considerando significativas displasia em pelo menos duas linhagens hemopoéticas, no sangue ou na medula óssea ,a

Hematologista, patologista clínica, médica assistente da Disciplina de Hematologia e Oncologia da Faculdade de Ciências Médicas da Santa Casa de São Paulo.

Correspondência: Teresa C Bortolheiro

Rua Marquês de Itu, 579 - $3^{\circ}$ andar - V. Buarque

01223-001 - São Paulo-SP

E-mail: bs.citologia@santacasasp.org.br; cbortolh@uol.com.br 
Tabela 1

A Classificação FAB

\begin{tabular}{|c|c|c|}
\hline Tiро & Sangue periférico & Medula óssea \\
\hline $\begin{array}{l}\text { Anemia Refratária } \\
\text { (AR) }\end{array}$ & Blastos $\leq 1 \%$ & Blastos $\leq 5 \%$ \\
\hline $\begin{array}{l}\text { Anemia refratária } \\
\text { com sideroblastos } \\
\text { em anel (ARSA) }\end{array}$ & Blastos $\leq 1 \%$ & $\begin{array}{l}\text { Blastos } \leq 5 \% \\
\text { Sideroblastos em } \\
\text { anel }>15 \%\end{array}$ \\
\hline $\begin{array}{l}\text { Anemia refratária } \\
\text { com excesso de } \\
\text { blastos (AREB) }\end{array}$ & Blastos $<5 \%$ & $\begin{array}{l}\text { Blastos }>5 \% \text { e } \\
<20 \%\end{array}$ \\
\hline $\begin{array}{l}\text { Leucemia } \\
\text { mielomonocítica } \\
\text { crônica (LMMC) }\end{array}$ & $\begin{array}{l}\text { Blastos }<5 \% \\
\text { Monócitos }>1000 / \mathrm{mm}^{3}\end{array}$ & Blastos $<20 \%$ \\
\hline $\begin{array}{l}\text { Anemia refratária } \\
\text { com excesso de } \\
\text { blastos em } \\
\text { transformação } \\
\text { (AREB-T) }\end{array}$ & Blastos $\geq 5 \%$ & $\begin{array}{l}\text { Blastos entre }>20 \% \text { e } \\
<30 \% \text { ou com } \\
\text { bastonetes de Auer }\end{array}$ \\
\hline
\end{tabular}

presença ou ausência de sideroblastos em anel, o número de blastos no sangue e na medula óssea e a presença de bastonetes de Auer. Os pacientes são divididos em cinco subgrupos: anemia refratária (AR), anemia refratária com sideroblastos em anel (ARSA), anemia refratária com excesso de blastos (AREB), anemia refratária com excesso de blastos em transformação (AREB-T) e leucemia mielomonocítica crônica (LMMC). Os quatro primeiros subtipos são caracterizados pela presença de menos de $5 \%$ de blastos, havendo na ARSA pelo menos $15 \%$ de sideroblastos em anel. Na AREB há entre 5\%-20\% de blastos e, na AREB-T, 21\%-29\% de blastos ou qualquer número de blastos com bastonete de Auer, sendo considerada leucemia mielóide aguda quando há 30\% de blastos. Na LMMC, os critérios incluíam monócitos acima de $1000 / \mathrm{mm}^{3}$, aumento de monócitos na medula óssea e blastos entre 1\%-20\% na medula óssea. A definição de blastos tipo I (agranulares) e tipo II (com grânulos azurófilos) também foi feita na mesma publicação. A classificação FAB permite delinear claramente grupos de baixo risco (AR e ARSA), com sobrevida de quatro a cinco anos; risco intermediário (AREB), com sobrevida de aproximadamente um ano; e alto risco (AREB-T) com rápida transformação para LMA e sobrevida muito curta. Outras variantes de SMD, que não se encaixavam nos subgrupos da classificação FAB, foram descritas: SMD com medula hipocelular, SMD com medula hiperfibrótica, SMD secundária relacionada a tratamento quimio ou radioterápico (t-SMD). A classificação FAB foi bem aceita pelos clínicos e pelos patologistas e, embora houvesse inúmeros casos que não podiam ser classificados dentro dos parâmetros por ela propostos, foi extremamente útil nos últimos 25 anos, ajudando a estabelecer abordagens diagnósticas e terapêuticas e a uniformizar a linguagem usada em todo mundo. Com o tempo, verificou-se que um dos maiores problemas era o grande número de blastos na AREB e na LMMC, aproximando-as das LMA,sendo também evidente que a LMMC apresentava características tanto de SMD quanto de doenças mieloproliferativas crônicas (DMPC). Posteriormente, o grupo FAB separou as LMMC em dois subgrupos, proliferativa (com leucócitos acima de $12.000 / \mathrm{mm}^{3}$, presença de hepatoesplenomegalia e sintomas clínicos) e não-proliferativa (com número menor de leucócitos e sem sintomas clínicos). Outros problemas incluíam: o uso do termo anemia refratária em casos em que as citopenias eram outras que não a anemia, a definição da síndrome do $5 q$-, e o fato de não se levar em conta a intensidade e o número das citopenias mostra ter grande importância na evolução da doença. A presença dos bastonetes de Auer isoladamente não mostrou estar relacionada a um pior prognóstico. ${ }^{7}$ Outra falha da classificação FAB é não considerar a intensa heterogeneidade das SMD, resultando em $5 \%-10 \%$ de casos que não se encaixam em nenhuma das categorias. ${ }^{8} \mathrm{O}$ surgimento do índice prognóstico internacional (IPSS) complementou a classificação FAB, adicionando parâmetros relevantes para o prognóstico como a citogenética, número de citopenias e idade dos pacientes.

\section{A classificação OMS}

Em 1997, um grupo de morfologistas e clínicos, 5,6,11 reunidos sob os auspícios da Organização Mundial da Saúde (OMS), iniciou os estudos para uma nova classificação das SMD. A classificação da OMS associa a imunofenotipagem e a genética aos parâmetros clínicos, morfológicos e citoquímicos utilizados na classificação FAB, e tem sido refinada constantemente com base em estudos clínicos e laboratoriais. A maior alteração proposta pela classificação da OMS, e também a mais controversa, é a diminuição do número de blastos para LMA de 30\% para 20\% , desaparecendo assim a categoria AREB-T, considerada por muitos especialistas como tendo sobrevida e resposta ao tratamento semelhante às das LMA. Entretanto, outros grupos consideram essa afirmação biologicamente inconsistente e apontam inúmeras diferenças entre AREB-T e LMA e sugerem a manutenção da categoria. Na AREB-T, por exemplo, são descritos índices altos de apoptose, semelhantes aos encontrados nos outros subtipos de SMD, mas não nas LMA, além de alterações citogenéticas complexas e mecanismos de resistência a drogas, que não são comumente vistos nas LMA de novo. ${ }^{2}$ Há também dados que mostram que a AREB-T tem pior resposta ao tratamento quimioterápico e pior prognóstico que as LMA de novo, assemelhando-se mais às LMA secundárias. ${ }^{9,10}$ Novos estudos são necessários para esclarecer esse ponto, sendo evidente que apenas o número de blastos é insuficiente para classificar a doença como agressiva ou indolente e para estabelecer o melhor tratamento. ${ }^{9,10}$ A AREB foi dividida em dois grupos: AREB -1, com blastos 5\%-9\%, e AREB-2, com blastos entre $10 \%-19 \%$. Nos casos com menos de $5 \%$ de blastos, devem ser distinguidos pacientes com displasia apenas na linhagem eritroblástica e os com displasia multilinhagem, que comprovadamente têm impacto no prog- 
Tabela 2

Classificação OMS

\begin{tabular}{|c|c|c|}
\hline Tipo & Sangue Periférico & Medula óssea \\
\hline Anemia Refratária (AR) & Anemia, blastos $<1 \%$ & $\begin{array}{l}\text { Displasia apenas na linhagem } \\
\text { eritroblástica, }<5 \% \text { de blastos }\end{array}$ \\
\hline $\begin{array}{l}\text { Anemia refratária com } \\
\text { sideroblastos em anel } \\
\text { (ARSA) }\end{array}$ & $\begin{array}{l}\text { Anemia } \\
\text { Ausência de blastos }\end{array}$ & $\begin{array}{l}\text { Displasia apenas na linhagem } \\
\text { eritroblástica, }<5 \% \text { de blastos, } \\
<15 \% \text { de sideroblastos } \\
\text { em anel }\end{array}$ \\
\hline $\begin{array}{l}\text { Citopenia refratária com } \\
\text { displasia Multilinhagem } \\
\text { (CRDM) }\end{array}$ & $\begin{array}{l}\text { Bi ou pancitopenia, } \\
<1 \% \text { blastos }\end{array}$ & $\begin{array}{l}\text { Displasia em } \geq 10 \% \text { das } \\
\text { células de duas ou mais } \\
\text { linhagens, }<5 \% \text { de blastos }\end{array}$ \\
\hline $\begin{array}{l}\text { Citopenia refratária com } \\
\text { displasia multilinhagem e } \\
\text { sideroblastos em anel } \\
\text { (CRDMSA) }\end{array}$ & $\begin{array}{l}\text { Bi ou pancitopenia, } \\
<1 \% \text { blastos }\end{array}$ & $\begin{array}{l}\text { Displasia em } \geq 10 \% \text { das } \\
\text { células de duas ou mais } \\
\text { linhagens, }<5 \% \text { de blastos } \\
\text { e } \geq 15 \% \text { de sideroblastos } \\
\text { em anel }\end{array}$ \\
\hline $\begin{array}{l}\text { Anemia refratária com } \\
\text { excesso de blastos }-1 \\
\text { (AREB-1) }\end{array}$ & $\begin{array}{l}\text { Bi ou pancitopenia, } \\
\text { blastos }<5 \%\end{array}$ & $\begin{array}{l}\text { Displasia uni ou multilinhagem } \\
5 \%-9 \% \text { de blastos }\end{array}$ \\
\hline $\begin{array}{l}\text { Anemia refratária com } \\
\text { excesso de blastos }-2 \\
\text { (AREB-2) }\end{array}$ & $\begin{array}{l}\text { Bi ou pancitopenia, } \\
\text { blastos } 5 \%-19 \% \\
\text { Monócitos }<1000 / \mathrm{mm}^{3}\end{array}$ & $\begin{array}{l}\text { Displasia uni ou multilinhagem } \\
10 \%-19 \% \text { de blastos }\end{array}$ \\
\hline $\begin{array}{l}\text { Síndrome mielodisplásica } \\
\text { inclassificável }\end{array}$ & $\begin{array}{l}\text { Neutropenia ou } \\
\text { plaquetopenia, Blastos } \\
\text { raros ou ausentes }\end{array}$ & $\begin{array}{l}\text { Displasia unilinhagem, } \\
\text { blastos }<5 \%\end{array}$ \\
\hline $\begin{array}{l}\text { Síndrome mielodisplásica } \\
\text { com del }(5 q) \text { isolada }\end{array}$ & $\begin{array}{l}\text { Anemia, plaquetas } \\
\text { normais ou elevadas, } \\
<5 \% \text { blastos }\end{array}$ & $\begin{array}{l}\text { Megacariócitos em número } \\
\text { normal ou elevado com } \\
\text { núcleos unilobulados } \\
<5 \% \text { blastos, } 5 q-\end{array}$ \\
\hline
\end{tabular}

\section{Conclusões}

Classificações baseadas na morfologia, como a FAB e OMS, embora imperfeitas, trouxeram grandes avanços para o diagnóstico e caracterização das SMD e devem servir como ponto de partida para futuros refinamentos, baseados em observações clínicas e novas técnicas diagnósticas. Futuros estudos possibilitarão adicionar novos parâmetros como a citometria de fluxo e análises moleculares, permitindo melhor avaliação clínica e biológica das SMD e a separação dos pacientes em grupos mais homogêneos, permitindo buscar terapêuticas mais específicas.

É preciso salientar que a presença de mielodisplasia não é, por si só, evidência do diagnóstico de síndrome mielodisplásica, podendo ser observada em grande número de doenças infecciosas, inflamatórias, neoplásicas, deficiência de vitaminas e uso de fatores de crescimento.

A classificação da OMS pode ser vista como uma evolução da classificação FAB e como ponto de partida para futuros estudos para melhor compreensão das SMD.

nóstico. A ARSA também foi dividida em duas categorias: com ou sem displasia multilinhagem, definida como displasia em mais de $10 \%$ das células de cada linhagem.

A síndrome do 5q- foi colocada separadamente por constituir um grupo de extremamente bom prognóstico e características bem definidas, como número de plaquetas elevado ou normal e a presença de megacariócitos mononucleares. A LMMC, por suas características clínicas e biológicas foi retirada da classificação de SMD e colocada numa nova categoria: doenças mieloproliferativas/mielodisplásicas. Casos que não se encaixam nas diversas categorias são colocados no subtipo SMD inclassificável. Muitos grupos têm feito avaliações críticas da classificação da OMS, com graus variáveis de concordância. ${ }^{12,1415}$ Germing et al. ${ }^{12}$ analisaram retrospectivamente 1.600 pacientes mantidos no registro da universidade de Dusseldorf, diagnosticados como SMD entre 1970-1999 conforme a classificação FAB, re-classificandoos segundo os critérios da OMS. Os resultados mostraram que a nova classificação é capaz de identificar mais adequadamente grupos de pior prognóstico, principalmente pelo reconhecimento da importância da displasia multilinhagem e da homogeinização de categorias antes heterogêneas, como as AR. Outros grupos, como do Instituto Ludwig de Viena, não conseguiram reproduzir os mesmos resultados. ${ }^{14}$
Enquanto aguardamos o refinamento da classificação da OMS através de sua correlação com aspectos clínicos e biológicos da doença, recomenda-se utilizar as duas classificações (Tabela 3)

Tabela 3

Correlação entre as classificações FAB e OMS

\begin{tabular}{ll}
\hline \multicolumn{1}{c}{ FAB } & \multicolumn{1}{c}{ OMS } \\
\hline $\begin{array}{l}\text { Anemia refratária(AR) } \\
<\% \text { blastos }\end{array}$ & $\begin{array}{l}\text { Anemia refratária (AR) } \\
\text { Citopenia refratária com } \\
\text { displasia multilinhagem } \\
\text { SMD Inclassificável }\end{array}$ \\
& SMD com del (5q) \\
Anemia refratária com & Anemia refratária com \\
sideroblastos em anel & sideroblastos em anel \\
$<5 \%$ blastos $+>15 \%$ & Anemia refratária com \\
sideroblastos em anel & sideroblastos em anel \\
& e displasia multilinhagem \\
Anemia refratária com & Anemia refratária com excesso \\
excesso de blastos & de blastos -1 (AREB-1) \\
(AREB) 5-20\% blastos & $5-9 \%$ blastos \\
& Anemia refratária com excesso \\
& de blastos-2 (AREB-2) \\
& $9-19 \%$ blastos \\
& Leucemia mielóide aguda \\
AREB em transformação & $\geq 20 \%$ blastos \\
\hline
\end{tabular}




\section{Abstract}

The initial classification of the myelodisplastic syndromes (MDS) was compiled in 1976 by the FAB group and was based on morphological parameters observed in the peripheral blood and in the bone marrow. The FAB classification was revised in 1982 and has been used in the last 25 years as a guide for a better understanding of this heterogeneous group of diseases. In 2001, the WHO published a new classification with significant modifications in the diverse subgroups of FAB with the intention of obtaining a better grouping of the subtypes with similar clinical behaviors. The most important modification was the decrease in the minimum number of blasts for the AML diagnosis from $30 \%$ to $20 \%$, resulting in the disappearance of the subtype RAEB-T. This is also the most polemic alteration, as there are innumerable publications discussing the evident clinical and biological differences between the MDS and $A M L$, with the opinion that the number of blasts alone is insufficient for the choice of therapy being unanimous. Another important point was the differentiation of the groups with dysplasia in single and multiple strains, which has been demonstrated as having great importance in the prognosis. Several studies have been published comparing the FAB and WHO classifications, recognizing the great contribution of the FAB classification for the better understanding of the MDS, as well as its shortcomings, and attempting to validate the modifications proposed by the WHO classification and identify the points liable to modification. Rev. bras. hematol. hemoter. 2006;28(3):194-197.

Key words: Myelodisplastic syndrome; morphological classification; dysplasia; prognostic factors.

\section{Referências Bibliográficas}

1. Greenberg PL. Clinical and prognostic characterization of myelodisplastic syndromes. In Myelodysplastic syndromes clinical and biological advances, $1^{\text {st }}$ ed, ed P. Greenberg. Cambridge, 2006, pp 1-24.

2. Brunning R. Morphologic classification of myelodysplastic syndromes: French-American-British (FAB) and World Health Organization (WHO). in Myelodysplastic syndromes clinical and biological advances, $1^{\text {st }}$ ed, ed P. Greenberg, Cambridge, 2006, pp 33-62.

3. Bennett JM, Catovsky D, Daniel MT, Flandrin G, Galton DAG, Gralnick HR, et al. Proposals for the classification of the myelodysplastic syndromes. Br J Haematol 1982;51:189.

4. Bennett JM, Catovsky D, Daniel MT, Flandrin G, Galton DAG, Gralnick HR, et al. Proposed revised criteria for the classification of acute myeloid leukemia. Ann Intern Med 1985;103:626-629.

5. Harris NL, Jaffe ES, Diebold J, Flandrin G, Muller-Hermelink HK, Vardiman J, et al. Word Health Organization classification of neoplastic diseases of the hematopoietic and lymphoid tissues: report of the clinical advisory committee meeting -Airlie House, Virginia, November 1997. J Clin Oncol 1999;17:3.835-3.849.

6. Mijovic A, Mufti GJ. The myelodysplastic syndromes: towards a functional classification. Blood Rev 1999;23:283-287.

7. Seymour JF, Estey EH. The prognostic significance of Auer rods. Brit J Haematol 1993;85:67-71.

8. Verhoef GEG, Pittaluga S, De Wolf-Peeters C, Boogaerts MA. FAB classification of myelodysplastic syndromes: merits and controversies. Ann Hematol 1995;71:3-7.
9. Albitar M, Beran M, O’Brian S, Kantagian H, Frieriech E, Keating $\mathrm{M}$, et al. Differences between refractory anemia with excess blasts and acute myeloid leukemia. Blood 2000;96;1:372-373.

10. Estey E, Thall P, Beran M, et al. Effect of diagnosis (refractory anemia with excess of blasts, refractory anemia with excess blasts in transformation, or acute myeloid leukemia [AML]) on outcome of AML-type chemotherapy. Blood 1997;90:2.969-2.977.

11. Harris NL, Jaffe ES, Diebold J, et al. World Health Organization classification of neoplastic diseases of the hematopoietic and lymphoid tissues: report of the Clinical Advisory Committee Meeting - Airlie House, Virginia, November 1997. J Clin Oncol 1999; $17: 3.835-3.849$

12. Germing U, Gattermann N, Strupp C, Aivado M, Aul C. Validation of the WHO proposals for a new classification of primary myelodysplastic syndromes: a retrospective analysis of 1600 patients. Leuk Res 2000;24:983-992

13. Bowen D, Culligan D, Jowitt S, Kelsey S, Mufti G, Oscier D, et al. The UK MDS guidelines group. Guidelines for the diagnosis and therapy of adult myelodysplastic syndromes. Brit J Haematol 2003; 120:187-200

14. Nosslinger T, Reisner R, Koller E, et al. Myelodysplastic syndromes, from French-American-British to World Health Organization: comparison of classifications on 431 unselected patients from a single institution. Blood 2001;98:2.935-2.941.

15. Howe RB, Porwit-MacDonald A, Wanat R, Tehranchi R, HellstromLindberg E. The WHO classification of MDS does make a difference. Blood 2004;103:3.265-3.270.

Avaliação: Um revisor externo e editorial board

Conflito de interesse: Não declarado

Recebido: 14/03/2006

Aceito após modificações: 11/09/2006 